\title{
ANÁLISIS PSICOMÉTRICO DE LA ESCALA AUTOEFICACIA EN CONDUCTAS ACADÉMICAS EN UNIVERSITARIOS DE PRIMER INGRESO
}

FIRST GRADE UNIVERSITY STUDENT'S PSYCHOMETRIC ANALYSIS OF AN SELFEFFICACY SCALE IN ACADEMIC BEHAVIOR

Volumen 11, Número 3

Setiembre-Diciembre

pp. 1-27

Este número se publicó el 15 de diciembre de 2011

\author{
Humberto Blanco Vega \\ Manuel Martínez Marín \\ María del Carmen Zueck Enríquez \\ Gabriel Gastélum Cuadras
}

La revista está indexada en los directorios:

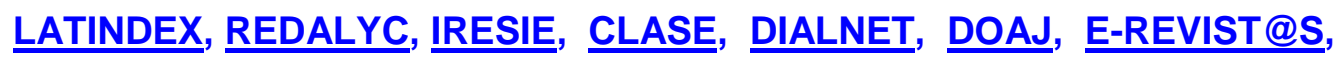

La revista está incluida en los sitios:

REDIE, RINACE, OEI, MAESTROTECA, PREAL, HUASCARAN, CLASCO 


\title{
ANÁLISIS PSICOMÉTRICO DE LA ESCALA AUTOEFICACIA EN CONDUCTAS ACADÉMICAS EN UNIVERSITARIOS DE PRIMER INGRESO FIRST GRADE UNIVERSITY STUDENT'S PSYCHOMETRIC ANALYSIS OF AN SELF- EFFICACY SCALE IN ACADEMIC BEHAVIOR
}

\author{
Humberto Blanco Vega ${ }^{1}$ \\ Manuel Martínez Marín ${ }^{2}$ \\ María del Carmen Zueck Enríquez \\ Gabriel Gastélum Cuadras ${ }^{4}$
}

\begin{abstract}
Resumen: El presente artículo tiene como propósito el diseño de la Escala Autoeficacia en Conductas Académicas (EACA) y el estudio de sus propiedades psicométricas desde la perspectiva del enfoque clásico de la teoría de los test y el de los modelos de ecuaciones estructurales, lo cual permitió realizar estudios paralelos para corroborar y verificar los resultados obtenidos. La muestra total fue de 2089 sujetos; 902 mujeres y 1187 hombres, estudiantes de primer ingreso a las licenciaturas que se ofrecen en la Universidad Autónoma de Chihuahua, con una edad media de 18.23 años $(D E=0.74)$. El análisis de las propiedades psicométricas, ha mostrado que una estructura trifactorial es viable y adecuada de acuerdo con los requisitos psicométricos establecidos cuando los informantes son los propios alumnos. Estos resultados nos llevaron a eliminar 14 de los 27 ítems analizados. La estructura de tres factores (Comunicación, Atención y Excelencia), atendiendo a criterios estadísticos y sustantivos, ha mostrado adecuados indicadores de ajuste, de fiabilidad y de validez. Además, los resultados de los análisis factoriales llevados a cabo con las submuestras 1 y 2, indican la existencia de fuertes evidencias de la estabilidad de la estructura factorial, hasta que no se demuestre lo contrario. Futuras investigaciones deberían replicar estos hallazgos en muestras más amplias.
\end{abstract}

Palabras clave: CONDUCTAS ACADÉMICAS, VALIDACIÓN ESCALAS, ANÁLISIS PSICOMÉTRICO, AUTOEFICACIA, MÉXICO

\begin{abstract}
This article focuses on the design of the Self-Efficacy Scale in Academic Behaviors (EACA) and on its psychometric properties from the classical test theory as well as the structural equation models; which allowed the development of parallel studies to verify the obtained results. The total sample comprised 2089 participants; 902 women and 1187 men, all of them freshman at Universidad Autónoma de Chihuahua, with a mean age of 18.23 years $(D S=0.74)$. Psychometric analysis showed that a three-factorial structure was viable and adequate according to the established psychometric requirements when the informers are the students themselves. Furthermore, results took us to eliminate 14 from the 27 analyzed items. The structure of the three factors (Communication, Attention and Excellence), attending to substantive and statistic criteria, showed adequate indicators of adjustment, reliability and validity. In addition, factor analysis conducted with subsamples 1 and 2 indicated the presence of strong evidence of factor structure stability, until proven otherwise. Future research should replicate these findings in larger samples.
\end{abstract}

Keywords: ACADEMIC BEHAVIORS, VALIDITY, SCALES, PSYCHOMETRIC ANALYSIS, SELF-EFFICACY, MEXICO

\footnotetext{
${ }^{\top}$ Doctor en Actividad Física y Salud por la Universidad de Granada. Licenciatura en Psicología Educativa y Maestría en Psicología Social y de las Organizaciones. Profesor de tiempo completo de la Universidad Autónoma de Chihuahua. Asignaturas impartidas en posgrado: Diseño de instrumentos, Estadística I y II. Dirección electrónica: hblanco@uach.mx

2 Doctor en Educación Física por la Universidad de Granada. Profesor Titular del Departamento de Educación Física y Deportiva de la Universidad de Granada. Secretario General de la Asociación Española de Ciencias del Deporte. Patentes: Pulsador electrónico de presión y Sistema de entrenamiento visual para lanzamiento de precisión. Dirección electrónica: manumar@ugr.es

${ }^{3}$ Doctora en Actividad Física y Salud por la Universidad de Granada. Profesora de tiempo completo de la Universidad Autónoma de Chihuahua. Asignaturas impartidas en posgrado: Diseño de instrumentos, Estadística l y II. Dirección electrónica: mzueck@uach.mx

${ }^{4}$ Doctor en Actividad Física y Salud por la Universidad de Granada. Profesor de tiempo completo de la Universidad Autónoma de Chihuahua. Asignaturas impartidas en posgrado: Diseño de instrumentos, Estadística I y II. Dirección electrónica: gastelum@uach.mx
}

Artículo recibido: 7 de junio, 2011

Aprobado: 10 de noviembre, 2011 


\section{Introducción}

Un ideal común a todas las personas es conseguir determinadas metas, la propia motivación impulsa al ser humano a emprender conductas específicas en función de los logros que éste pretende alcanzar. Mas no basta conocer con claridad aquello que deseamos lograr, ni tampoco el mejor medio para poder conseguirlo. Tampoco, basta con ser capaz de; es preciso juzgarse capaz de utilizar las capacidades y las habilidades personales ante circunstancias muy diversas. La percepción de las personas acerca de su propia eficacia se alza como un requisito fundamental para desarrollar con éxito las acciones conducentes al logro de los objetivos personales. Dicha autopercepción, denominada autoeficacia, ejerce una profunda influencia en la elección de tareas y actividades, en el esfuerzo y en la perseverancia de las personas cuando se enfrentan a determinados retos e incluso en las reacciones emocionales que experimentan ante situaciones difíciles (Prieto, 2003). En definitiva, las creencias de autoeficacia representan un mecanismo cognitivo que media entre el conocimiento y la acción y que determina, junto con otras variables, el éxito de las propias acciones (Prieto, 2003).

Dentro de los contextos educativos ha existido un interés permanente por comprender los factores cognitivos y comportamentales que favorecen o dificultan el desempeño del estudiante en sus labores académicas y cómo el desempeño se relaciona con su desarrollo integral. En el área de la psicología educativa, específicamente, el constructo de autoeficacia ha recibido especial atención y se han generado importantes avances de investigación que han contribuido al mejoramiento de prácticas pedagógicas y de enseñanza (Prieto, 2003). La investigación empírica ha demostrado, de manera amplia, que la autoeficacia resulta ser más predictiva del rendimiento académico que otras variables cognitivas (Bandura, 1982), también que logra predecir el éxito posterior (Bandura, 1997; Pajares y Schunk, 2001) y que es un importante mediador cognitivo de competencia y rendimiento (Valiante, 2000) en cuanto favorece los procesos cognoscitivos (Pintrich y De Groot, 1990).

Por todo ello, en este trabajo forma parte de un estudio más extenso, cuyo propósito general radica en determinar las diferencias y las similitudes entre los estudiantes de primer ingreso a las diferentes licenciaturas de la Universidad Autónoma de Chihuahua en cuanto a su autoeficacia percibida, comprensión lectora y habilidades cognitivas, entre otras; con la finalidad de contar con información valiosa para los sistemas de tutoría y desarrollo personal en nuestra universidad. Se analiza la consistencia interna y la estructura factorial de un 
instrumento de autoreporte que permite identificar conductas académicas, cuyo nivel de autoeficacia percibido por los alumnos de nuevo ingreso representen un área de oportunidad o mejora; en relación con el resto de los alumnos, aportando evidencias y datos que propicien la intervención educativa dentro de una perspectiva de atención a la diversidad en el aula. Para obtener un instrumento con las mejores propiedades psicométricas, se realizó el análisis en dos submuestras, desde la perspectiva del enfoque clásico de la teoría de los test (Muñiz, 1998; Nunnally y Bernstein, 1995) y el de los modelos de ecuaciones estructurales (Batista y Coenders, 2000); lo cual permitió realizar estudios paralelos para corroborar y verificar los resultados obtenidos.

\subsection{La autoeficacia como constructo}

La autoeficacia es tradicionalmente referida a un dominio o una tarea específica. Sin embargo, algunos investigadores también la han conceptualizado en una forma general de autoeficacia que se refiere a un sentido amplio y estable de competencia personal sobre cuán efectiva puede ser la persona al afrontar una variedad de situaciones estresantes (Choi, 2004; Luszczynska, Scholz y Schwarzer, 2005; Luszczynska, Gibbons, Piko y Tekosel, 2004).

Para la mayoría de las aplicaciones, Bandura $(1997,2001)$ plantea que la autoeficacia percibida debe ser conceptualizada de manera específica. La autoeficacia percibida hace, pues, referencia a las creencias de las personas acerca de sus propias capacidades para el logro de determinados resultados. Por consiguiente, el sistema de creencias de eficacia no es un rasgo global, sino un grupo de autocreencias ligadas a ámbitos de funcionamiento diferenciado (Bandura, 2001).

Bandura (2001) considera, por tanto, a las expectativas de autoeficacia no como variables globales y estables, sino como cogniciones específicas y cambiantes, que se forman y se reelaboran constantemente a partir del procesamiento y la integración de la información procedente de diferentes orígenes. Es decir, la autoeficacia se refiere a la confianza que tiene una persona de que posee la capacidad para hacer las actividades que trata de hacer; de ahí que los juicios que la persona hace acerca de su autoeficacia son específicos de las tareas y las situaciones en que se involucra, y las personas las utilizan para referirse a algún tipo de meta o tarea a lograr. Por ejemplo, Zimmerman (1995) define la autoeficacia, referida a la realización de las tareas propias de la escuela, como los juicios 
personales acerca de las capacidades para organizar y conductas que sirvan para obtener tipos determinados de desempeño escolar. En este sentido, la autoeficacia para la escuela es un concepto muy específico que se refiere a la evaluación que el estudiante hace con respecto a sus capacidades para realizar las actividades propias de la escuela.

Por lo que se puede definir el término autoeficacia como los juicios que cada individuo hace sobre sus capacidades, con base en los cuales organizará y ejecutará sus actos de modo que le permitan alcanzar el rendimiento deseado (Bandura, 1997), o como plantea Pajares (1996), las creencias que tiene la persona sobre sus capacidades para organizar y ejecutar caminos para la acción requeridos en situaciones esperadas o basadas en niveles de rendimiento, o Schunk y Zimmerman (1997) que la definen como las creencias que tiene la persona sobre sus capacidades para aprender o desempeñar comportamientos en niveles previamente definidos.

\subsection{Rendimiento académico y autoeficacia}

Se ha demostrado, suficientemente, que la autoeficacia, la autorregulación, y el dominio de estrategias de estudio efectivas son factores importantes en el rendimiento académico, junto a otros, tales como las aptitudes cognitivas, la responsabilidad en la tareas escolares, las aspiraciones educacionales de la familia y del propio estudiante, y la calidad de la enseñanza impartida (Pintrich y De Groot, 1990; Wolters, 2004; Zimmerman y Kitsantas, 2005).

La aplicación de la teoría de la autoeficacia de Bandura en el ámbito educativo muestra cómo los estudiantes con altas expectativas de autoeficacia gozan de mayor motivación académica. Asimismo, obtienen mejores resultados, son más capaces de autorregular eficazmente su aprendizaje y muestran mayor motivación intrínseca cuando aprenden (Bandura, 1997). En consecuencia, la mejora de las expectativas de autoeficacia incrementa la motivación y el rendimiento en las tareas de aprendizaje.

Por tanto, las creencias que tienen las personas sobre sí mismas representan un factor básico para el logro de sus actividades o la toma de decisiones que enfrentarán a lo largo de su vida. Cuanto mayor sea la eficacia percibida, mayor el grado de esfuerzo que se invierta y mayor también la persistencia en el logro de la meta propuesta; situación de suma importancia para que una persona que se encuentra en un proceso de aprendizaje tenga éxito (Bandura, 1997). 
En el contexto educativo, Pajares (2002b) indica que el papel mediacional de la autoeficacia en la conducta se desarrolla a partir de la experiencia de dominio (los estudiantes que obtienen calificaciones altas desarrollan un sentido fuerte de confianza de sus capacidades en dicha área), la experiencia delegada (efectos producidos por las acciones de otros), las persuasiones sociales (mensajes que se reciben de otros favoreciéndolas creencias de autoeficacia), y los estados fisiológicos asociados con la ansiedad, la tensión, la excitación, la fatiga y los estados de ánimo (los individuos estiman su confianza, en parte, por el estado emocional que experimentan cuando realizan una acción).

De esta manera, las creencias de autoeficacia ejercen influencia en la percepción de los estudiantes sobre su capacidad para desempeñar las tareas requeridas en la elección y las metas propuestas, y en el esfuerzo y persistencia de las acciones encaminadas para lograr dicha meta, fomentando patrones de pensamiento y reacciones emocionales favorables (Bandura, 2000; Pintrich y García, 1993). La autoeficacia repercute también en la motivación de tal manera que los estudiantes se comprometen en aquellas tareas en las que se sienten más competentes y seguros y esto, a su vez, depende de sus propios juicios acerca de lo que ellos creen que pueden lograr (Bandura, 1986). En general, se puede afirmar que a más alta percepción de competencia, mayores exigencias, aspiraciones y dedicación (Bong, 2001; Huertas, 1997). Cabe señalar que en la teoría de Bandura se reconoce de forma explícita que las expectativas no agotan los determinantes de la conducta: para que se produzca una buena actuación en un ámbito determinado, además de unas altas expectativas de eficacia y de resultados, se necesita poseer, como mínimo, las habilidades y los recursos materiales necesarios.

En el ambiente escolar, las teorías actuales que estudian el aprendizaje y la instrucción postulan que los estudiantes son buscadores y procesadores activos de información (Pintrich, Cross, Kozma, y McKeachie, 1986). Las investigaciones indican que las cogniciones de los estudiantes influyen en la instigación, dirección, fuerza y persistencia de sus conductas dirigidas al logro (Schunk, 1989; Weinstein, 1989; Zimmerman, 1990). Sin embargo, hay factores que afectan el rol mediacional que los juicios de autoeficacia tienen en el comportamiento. Nos referimos a la falta de incentivos y a otras limitaciones. Es decir, una persona con una alta autoeficacia y habilidad puede decidir no comportarse de acuerdo con sus creencias y habilidades. Esto se explica porque puede carecer de los recursos necesarios para hacerlo, de incentivos o puede encontrar restricciones sociales en sus 
deseos. En estos casos, la autoeficacia fallará en la predicción del comportamiento. De igual manera, en situaciones donde las metas y el desempeño necesario para lograrlas no son claros, la autoeficacia percibida es de poca utilidad para predecir el comportamiento. Las personas no saben cuánto esfuerzo emplear, cuánto tiempo sostenerlo, cómo corregir errores, etc. En estas situaciones, los sujetos no pueden evaluar eficazmente su autoeficacia y deben confiar en experiencias previas. Esto ofrece una pobre predicción del desempeño (Pajares, 2002a).

Como una muestra de la importancia de la autoeficacia en el ámbito académico, podemos decir que ésta revela por qué las personas con el mismo nivel de habilidad y conocimiento presentan conductas y/o resultados diferentes, o por qué las personas actúan en disonancia con sus habilidades (Pajares, 2002a). Lo anterior se explica porque el rendimiento académico adecuado también depende de la eficacia percibida para manejar demandas académicas exitosamente. Por ello, las creencias de autoeficacia en la propia capacidad son imprescindibles para dominar las actividades académicas, ya que los estudiantes que confían en sus capacidades se sienten más motivados para alcanzar sus metas. De igual modo, las personas que dudan de sus capacidades pueden creer que las cosas son más difíciles de lo que realmente son, creencia que genera tensión, depresión y una visión estrecha para resolver problemas (Pajares y Schunk, 2001). Se ha evidenciado que un bajo nivel de autoeficacia puede ser responsable no sólo de disminución del rendimiento escolar e interés hacia el estudio, sino también de comportamientos inadaptados en jóvenes (Hackett, 1995), de ahí la importancia de que la educación fortalezca el desarrollo de la competencia académica en el estudiante y fomente habilidades que le permitan creer en sus propias capacidades (Pajares, 2001).

Por todo ello, se puede concluir que la autoeficacia tiene un papel vital en el ámbito académico, de ahí que un buen desempeño académico no puede ser garantizado solo por los conocimientos y la habilidad de los individuos. Las creencias de eficacia pueden determinar un desempeño diferente en dos personas con el mismo grado de habilidad. Esto se debe a que el éxito académico demanda procesos reguladores como la autoevaluación, el automonitoreo y el uso de estrategias metacognitivas de aprendizaje, procesos que son influidos positivamente por un alto grado de creencia en la propia capacidad o autoeficacia (Pajares y Schunk, 2001). Asimismo, la autoeficacia académica representa un factor de vital importancia si se quiere formar personas que aprendan de por vida, ya que las creencias en 
las propias capacidades para manejar actividades académicas afectan el nivel de aspiración de los estudiantes, su preparación para diferentes carreras, además de su nivel de interés de logros intelectuales y sus éxitos académicos (Bandura, 1995). Además, en relación con la motivación académica, las creencias de autoeficacia afectan el nivel de esfuerzo, persistencia y la elección de actividades. Alumnos con un elevado sentido de eficacia para cumplir tareas educativas persistirán más ante dificultades trabajarán con más intensidad y participarán más que aquellos que duden de sus capacidades (Bandura, 1995).

\subsection{Escalas para la medición de autoeficacia}

La revisión de la literatura muestra que los instrumentos más utilizados para medir el constructo de autoeficacia son escalas de autorreporte. Dentro de éstas podemos distinguir dos grupos: en el primer grupo se encuentran aquellas escalas que miden un sentido de eficacia específico. En relación con este tipo de medición, Bandura $(1997,2001)$ plantea que es importante que las escalas se ajusten al dominio particular de funcionamiento que es objeto de interés. Entre las escalas desarrolladas dentro de esta línea está la Escala de Autoeficacia Percibida para Niños, que es una escala multidimensional que mide 7 distintos dominios específicos de la autoeficacia en niños y preadolescentes, como por ejemplo, autoeficacia para el logro académico y la autoeficacia en habilidades asertivas (Pastorelli et al., 2001). En el segundo grupo están las escalas que miden un sentido general de autoeficacia. Dentro de éstas se puede destacar la Escala Generalizada de Autoeficacia (Luszczynska et al., 2005). Esta escala ha sido traducida a 25 idiomas y utilizada en diversas culturas, en población adulta y adolescente desde los 12 años de edad.

\section{Método}

\subsection{Sujetos}

La muestra de 2089 sujetos, 902 mujeres y 1187 hombres se obtuvo mediante un muestreo por conveniencia, tratando de abarcar la representatividad de las diferentes licenciaturas que se ofrecen en la Universidad Autónoma de Chihuahua; luego, fue aleatoriamente dividida en dos submuestras (50\% y 50\%) utilizando el Statistical Package for the Social Sciences (SPSS) en su versión 15.0 (Tabla N. ${ }^{\circ}$ 1); con el fin de realizar estudios paralelos que permitieran corroborar y verificar los resultados obtenidos (validación cruzada). 
La primera mitad (submuestra 1) quedó constituida por 1065 sujetos; 474 mujeres y 591 hombres. Las edades fluctúan entre los 17 y 20 años, con una media de 18.23 y una desviación estándar de 0.75 años. La segunda mitad (submuestra 2) quedó compuesta por 1024 sujetos; 428 mujeres y 596 hombres. Las edades fluctuaron entre los 17 y 20 años, con una media de 18.22 y una desviación estándar de 0.74 años.

Tabla $N^{\circ}$ 1. Distribución de los sujetos en la muestra y submuestras

\begin{tabular}{lccc}
\hline & \multicolumn{2}{c}{ Submuestra } & \\
\cline { 2 - 3 } \multicolumn{1}{c}{ Disciplina } & 1 & 2 & Total \\
\hline Educación Física & 163 & 132 & 295 \\
Ciencias de la Salud & 113 & 116 & 229 \\
Educación y Humanidades & 84 & 84 & 168 \\
Ciencias Sociales y Administrativas & 162 & 138 & 300 \\
Ciencias Políticas & 146 & 143 & 289 \\
Ingeniería y Tecnología & 283 & 309 & 592 \\
Ciencias Agropecuarias & 114 & 102 & 216 \\
Total & 1065 & 1024 & 2089 \\
\hline
\end{tabular}

\subsection{Instrumento}

Previo a la elaboración del instrumento, se realizó una consulta con docentes de la Universidad Autónoma de Chihuahua en relación con los aspectos a utilizar en la medición de las variables contempladas en el estudio; como resultado de la consulta se redactaron 27 ítems relacionados con conductas académicas del alumno. Cada uno de los ítems obtenidos tuvieron que ser corregidos de tal manera que los sujetos pudieran contestarlos en tres escenarios distintos: actual, ideal y de cambio, diseñando así una encuesta tipo Likert, asistida por computadora, donde el encuestado responde a cada uno de los 27 ítems, en una escala de 0 a 10, la frecuencia con que, actualmente, en forma ideal y si se esfuerza en cambiar, realizaría o manifestaría una acción. Se eligió este tipo de encuesta por ser fácil de construir y de aplicar; además, de proporcionar una buena base para una primera ordenación de los individuos en la característica que se mide; en nuestro caso autoeficacia (Nunnally y Bernstein, 1995).

Aún cuando cada sujeto respondió a los 27 ítems del instrumento en tres escenarios distintos: Escenario actual, respondiendo en el contexto: actualmente con que frecuencia realiza determinada conducta o se percibe con determinada característica. Escenario ideal, 
respondiendo en el contexto: con que frecuencia desearía realizar determinada conducta o percibirse con determinada característica. Escenario de cambio, respondiendo en el contexto: si me esfuerzo en cambiar con que frecuencia realizaría determinada conducta o me percibiría con determinada característica. En el análisis psicométrico solo se utilizaron las respuestas al primer escenario.

\subsection{Procedimiento}

Se invitó a participar en el estudio a los alumnos de primer ingreso de las licenciaturas que se ofrecen en la Universidad Autónoma de Chihuahua; quienes aceptaron participar firmaron la carta de aceptación correspondiente. Luego, se aplicó el instrumento antes descrito por medio de una computadora personal y utilizando el módulo administrador del instrumento del editor de escalas versión 2.0 (Blanco et al., 2007), en una sesión de aproximadamente 25 minutos; en los laboratorios o centros de cómputo de las unidades académicas participantes. Al inicio de cada sesión se hizo una pequeña introducción sobre la importancia de la investigación y de cómo acceder al instrumento; las instrucciones de cómo responder se encontraban en las primeras pantallas antes del primer reactivo del instrumento. Al término de la sesión se les agradeció su participación.

Una vez aplicado el instrumento se procedió a recopilar los resultados por medio del módulo generador de resultados del editor de escalas versión 2.0 (Blanco et al., 2007).

\section{Resultados}

\subsection{Análisis clásico de las propiedades psicométricas de la escala}

Para establecer las dimensiones subyacentes (factores) de la escala, se realizaron sendos análisis factoriales exploratorios con las submuestras 1 y 2 ; después, mediante el coeficiente $\alpha$ de cronbach se estimó la consistencia interna para cada factor retenido como una medida de su fiabilidad (Nunnally y Bernstein, 1995).

\subsubsection{Análisis factorial exploratorio (primera solución factorial)}

Para determinar el número mínimo de factores comunes capaces de reproducir, de un modo satisfactorio, las correlaciones observadas entre los 27 ítems del instrumento empleado, se realizó, en la submuestra 1, la extracción de éstos a partir del método de componentes principales, tomando como base el criterio de Kaiser-Guttman (1958), por el 
que se toman autovalores mayores o igual a la unidad y el análisis gráfico del Scree Test de Cattell (1966) sobre los autovalores; además, para garantizar una adecuada representación de las variables (ítems), solo se conservaron aquéllos cuya comunalidad, o proporción de su varianza explicada por la solución factorial, fuera superior a .45. Este primer análisis puso de manifiesto una estructura factorial de tres componentes y nos llevó a eliminar 12 de los 27 ítems analizados (Tabla N. ${ }^{\circ}$ 2). Previamente, la significación del Test de Barlett (10684.232; $\mathrm{p}<0.0001)$ y el KMO (.927) mostraron una adecuada correlación entre los ítems y una muy buena adecuación muestral respectivamente.

Tabla $\mathbf{N}^{\circ} 2$

Ítems eliminados. Primera solución factorial. Análisis factorial exploratorio submuestra 1

\begin{tabular}{l} 
Ítems \\
\hline 6 Intento comprender el porqué de las cosas. \\
$9 \mathrm{El}$ nivel de respuesta que doy en mis exámenes corresponde al que mis profesores \\
pretenden que logre. \\
11 Cuando no estoy de acuerdo con alguna calificación, planteo mis argumentos \\
correctamente. \\
12 Aporto mis propias ideas en los trabajos que realizo. \\
13 Al realizar mis trabajos hago consultas bibliográficas extras a las que son \\
sugeridas. \\
18 Proporciono apoyo o soy cooperador con mis compañeros. \\
20 Cuando alguno de mis profesores comete un error soy capaz de indicárselo. \\
21 Leo revistas científicas donde se reportan investigaciones relacionadas con mi \\
carrera. \\
22 Envío señales a la gente a través de mis ojos y gestos corporales para que sepa \\
que estoy escuchando. \\
23 Soy capaz de relajarme al comunicarme con los demás de tal manera que ellos \\
se sientan cómodos al añadir sus propios comentarios. \\
24 Mi concentración es adecuada al escuchar a los demás. \\
25 Por lo general, pido a las personas que aclaren lo que han dicho en lugar de \\
adivinar el significado.
\end{tabular}

Sobre el conjunto de los 15 ítems obtenidos en el análisis previo, se realizó de nuevo un análisis de componentes principales para el conjunto de la submuestra 1; siguiendo el criterio de Kaiser (1958) y el análisis gráfico del Scree Test de Cattell (1966) sobre los autovalores, detectándose, después de una rotación varimax (Costello y Osborne, 2005), 
una estructura de tres factores: Comunicación con un $20.11 \%$ de la varianza explicada, Atención con un $19.64 \%$ de la varianza y Excelencia con un $18.68 \%$. El conjunto de los componentes principales seleccionados explicaron el $58.43 \%$ de la varianza (Tablas $\mathrm{N} .{ }^{\circ} 3 \mathrm{y}$ 4). La significación del Test de Barlett (6272.441; $p<0.0001)$ y el KMO (.877) muestran, además, una adecuada correlación entre los ítems y una buena adecuación muestral respectivamente.

Con el fin validar la solución factorial obtenida con la submuestra 1, se realizó nuevamente un análisis de componentes principales con los datos de la submuestra 2; siguiendo los mismos criterios para la retención de factores; encontrándose de nuevo, después de una rotación varimax (Costello y Osborne, 2005), una estructura de tres factores o componentes: Comunicación con un $20.08 \%$ de la varianza explicada, Atención con un $20.07 \%$ de la varianza y Excelencia con un 18.92\%. El conjunto de los componentes principales seleccionados explicaron el $59.07 \%$ de la varianza (Tabla N. ${ }^{\circ} 3$ y 4 ). La significación del Test de Barlett (6115.678; p< 0.0001) y el KMO (.884) muestran, además, una adecuada correlación entre los ítems y una buena adecuación muestral respectivamente.

Tabla $N^{\circ} 3$. Autovalores y porcentaje de varianza explicada por los factores retenidos. Análisis factorial exploratorio Submuestras 1 y 2 . Soluciones rotadas

\begin{tabular}{lccccccc}
\hline & \multicolumn{3}{c}{ Submuestra 1 } & & \multicolumn{3}{c}{ Submuestra 2 } \\
\cline { 2 - 4 } \multicolumn{1}{c}{ Factor } & Autovalor & \% varianza & \% acumulado & & Autovalor & \% varianza & \% acumulado \\
\hline 1. Comunicación & 3.02 & 20.11 & 3.02 & & 3.01 & 20.08 & 20.08 \\
2. Atención & 2.95 & 19.64 & 2.95 & & 3.01 & 20.07 & 40.15 \\
3. Excelencia & 2.80 & 18.68 & 2.80 & & 2.84 & 18.92 & 59.07 \\
\hline
\end{tabular}


Tabla $\mathbf{N}^{\circ}$ 4. Ítems agrupados por factor. Análisis factorial exploratorio Submuestras 1 y 2 . Soluciones rotadas

\begin{tabular}{|c|c|c|c|c|c|c|c|}
\hline \multirow{2}{*}{\multicolumn{2}{|c|}{ Ítem }} & \multicolumn{3}{|c|}{ Submuestra 1} & \multicolumn{3}{|c|}{ Submuestra 2} \\
\hline & & 1 & 2 & 3 & 1 & 2 & 3 \\
\hline 4 & Expreso mis ideas con claridad & .73 & & & .76 & & \\
\hline 5 & Hago comentarios y aportaciones pertinentes & .66 & & & .69 & & \\
\hline 19 & $\begin{array}{l}\text { En caso de desacuerdo soy capaz de entablar un } \\
\text { diálogo con mis profesores }\end{array}$ & .72 & & & .67 & & \\
\hline 26 & $\begin{array}{l}\text { Me siento bien con mi propio desempeño cuando } \\
\text { hablo enfrente de una clase o grupo de gente }\end{array}$ & .80 & & & .80 & & \\
\hline 27 & $\begin{array}{l}\text { Me siento cómodo al hablar frente a un grupo } \\
\text { numeroso de personas }\end{array}$ & .82 & & & .80 & & \\
\hline 2 & $\begin{array}{l}\text { Escucho con atención cuando el profesor aclara } \\
\text { una duda a un compañero }\end{array}$ & & .76 & & & .75 & \\
\hline 3 & $\begin{array}{l}\text { Escucho con atención las preguntas y } \\
\text { aportaciones de mis compañeros }\end{array}$ & & .70 & & & .72 & \\
\hline 7 & $\begin{array}{l}\text { Pongo atención cuando los profesores dan la } \\
\text { clase }\end{array}$ & & .68 & & & .71 & \\
\hline 8 & $\begin{array}{l}\text { Pongo atención cuando un compañero expone en } \\
\text { clase }\end{array}$ & & .69 & & & .69 & \\
\hline 17 & $\begin{array}{l}\text { Escucho con atención las preguntas y } \\
\text { comentarios de mis profesores }\end{array}$ & & .70 & & & .68 & \\
\hline 1 & Cumplo con las tareas que se me asignan & & & .70 & & & .67 \\
\hline 10 & $\begin{array}{l}\text { Me preparo para mis exámenes apoyándome en } \\
\text { los apuntes de clase, el texto del curso y lecturas } \\
\text { adicionales }\end{array}$ & & & .57 & & & .52 \\
\hline & $\begin{array}{l}\text { Entrego puntualmente los trabajos que se me } \\
\text { encargan }\end{array}$ & & & .75 & & & .73 \\
\hline & Soy cumplido en cuanto a mi asistencia & & & .75 & & & .83 \\
\hline & Soy cumplido en cuanto a mi puntualidad & & & .70 & & & .76 \\
\hline
\end{tabular}

\subsubsection{Congruencia entre los factores de las dos soluciones factoriales (validación cruzada)}

Los valores de los coeficientes de congruencia y de los coeficientes de correlación de Pearson entre los pesos factoriales de los componentes obtenidos en los análisis factoriales exploratorios llevados a cabo con las submuestras 1 y 2 ; indican, de acuerdo con lo sugerido por Cureton y D'Agostino (1983), Mulaik (1972) y Cliff (1966), una alta congruencia entre pares de componentes (Tabla N. ${ }^{\circ}$ ). 
Tabla N.$^{\circ}$ 5. Coeficientes de congruencia y de correlación de Pearson entre pesos factoriales. Análisis factoriales exploratorios submuestras 1 y 2

\begin{tabular}{lcc}
\hline \multicolumn{1}{c}{ Factor } & $\begin{array}{c}\text { Coeficiente de } \\
\text { congruencia }\end{array}$ & $\begin{array}{c}\text { Coeficiente de } \\
\text { correlación }\end{array}$ \\
\hline 1. Comunicación & .999 & .853 \\
2. Atención & .999 & .758 \\
3. Excelencia & .997 & .912 \\
\hline
\end{tabular}

\subsubsection{Fiabilidad de las subescalas (consistencia interna)}

Las subescalas (factores) resultantes en los análisis factoriales exploratorios, de ambas submuestras, poseen alfas superiores a .7, evidenciando una consistencia interna adecuada para este tipo de subescalas, particularmente si se considera el número reducido de ítems (Tabla N. $\left.{ }^{\circ} 6\right)$.

Tabla $\mathrm{N}^{\circ}{ }^{\circ}$ 6. Coeficientes de consistencia interna de los componentes obtenidos en los análisis factoriales exploratorios submuestras 1 y 2

\begin{tabular}{lcc}
\hline \multicolumn{1}{c}{ Factor } & $\alpha$ Submuestra 1 & $\alpha$ Submuestra 2 \\
\hline 1. Comunicación & .829 & .818 \\
2. Atención & .818 & .815 \\
3. Excelencia & .780 & .806 \\
\hline
\end{tabular}

\subsection{Análisis de las propiedades psicométricas de la escala mediante modelos de ecuaciones estructurales}

\subsubsection{Análisis factorial confirmatorio (primera solución factorial)}

Cuando se mide un único constructo, se hace necesario comprobar que se cumpla el requisito de la unidimensionalidad (Bagozzi y Baumgartner, 1994; Leighton, Gokiert y Cui, 2007), por la cual debe ser precisamente un único constructo el que subyace en un conjunto de ítems que constituyen una escala. Pero si hay algún constructo intrínsecamente multidimensional, como es el caso de la escala utilizada en este trabajo, la unidimensionalidad se ha de analizar para cada factor o dimensión individual. La unidimensionalidad de una variable se puede detectar mediante la realización de un análisis factorial exploratorio (Steenkamp y van Trijp, 1991; Leighton et al., 2007). 
En nuestro caso, se hizo a través de un análisis de ejes principales con rotación varimax (Hair et al., 1999), que permitió elegir los ítems que tienen una carga factorial alta en cada uno de los factores o componentes extraídos (Comunicación, Atención y Excelencia). Ahora, mediante el análisis factorial confirmatorio sobre los datos de la submuestra 2, se contrasta la solución factorial y se depura el conjunto de ítems resultantes del análisis factorial exploratorio realizado con los datos de la submuestra 1; con el fin de obtener modelos congenéricos $\mathrm{y}$, al mismo tiempo, probar la validez de constructo de las dimensiones y variables estudiadas. En el contexto de las ecuaciones estructurales, un modelo congenérico se caracteriza porque cada variable superficial (ítem) está conectada solamente con un factor, y las covariaciones se deben a las relaciones entre factores y variables superficiales (Catena et al., 2003).

Para conducir el análisis factorial confirmatorio, se utilizó el software AMOS 16 (Arbuckle, 2007), las varianzas de los términos de error fueron especificados como parámetros libres, en cada variable latente (factor) se fijó uno de los coeficientes estructurales asociados a uno, para que su escala se igual a la de una de las variables superficiales (ítems). El método de estimación empleado fue el de Máxima Verosimilitud (Hu y Bentler, 1995); siguiendo la recomendación de Thompson (2004), en el sentido de que cuando se emplea análisis factorial confirmatorio se debe corroborar no sólo el ajuste de un modelo teórico sino que es recomendable comparar los índices de ajuste de varios modelos alternativos para seleccionar el mejor.

Para evaluar el ajuste del modelo se siguieron las recomendaciones antes planteadas, que indican la conveniencia de emplear múltiples indicadores de ajuste (Hu y Bentler, 1995). Específicamente, se emplean el estadístico Chi-cuadrado, el índice de bondad de ajuste (GFI), el residuo cuadrático medio (RMSR), el error cuadrático medio de aproximación (RMSEA) y el índice de validación cruzada esperada (ECVI) como medidas absolutas de ajuste. El índice de bondad ajustado (AGFI), el Índice Tucker-Lewis (TLI), el Índice del ajuste normal (NFI) y el índice de bondad de ajuste comparativo (CFI) como medidas de ajuste incremental. El índice de ajuste normado de parsimonia (PNFI), el índice de calidad de ajuste de parsimonia (PGFI), la razón de Chi-cuadrado sobre los grados de libertad (CMIN/GL) y el Criterio de Información de Akaike (AIC) como medidas de ajuste de parsimonia.

De acuerdo con los resultados de la Tabla $\mathrm{N} .^{\circ} 7$, el análisis factorial confirmatorio inicial (15 ítems) indica que el modelo de medición de tres factores ajusta aceptablemente (GFI 
.912; RMSR 0.084; RMSEA .084; ECVI 0.760), pero no de manera óptima. Un examen de los coeficientes de regresión estandarizados de los ítems con cada uno de sus factores latentes permite identificar que los ítems 27 y 16 son los que menor valor poseen (.63 con el factor Comunicación y .57 con el factor Excelencia, respectivamente). Retirando estos ítems, los índices mejoran de manera considerable y el ajuste de los datos al modelo teórico de 13 ítems agrupados en tres factores es óptimo (GFI .973; RMSR 0.085; RMSEA .043; ECVI 0.234) y de acuerdo con las medidas de ajuste incremental y de parsimonia (Tablas 8 y 9 ) significativamente superior, al modelo alternativo de 15 ítems agrupados en tres factores.

\subsubsection{Análisis factorial confirmatorio (segunda solución factorial)}

Para comprobar la estructura factorial de la escala autoeficacia percibida en conductas académicas obtenidas a partir de la submuestra 2 (análisis factorial confirmatorio, primera solución factorial), se realizó un segundo análisis factorial confirmatorio en la submuestra 1. La estructura factorial analizada plantea un modelo de tres factores, con adscripciones de los ítems basados en análisis factorial confirmatorio de la submuestra 2 (3 factores 13 ítems). De acuerdo con los resultados de la Tabla No. 7, el segundo análisis factorial confirmatorio (submuestra 1) indica que el modelo de medición de tres factores es óptimo (GFI .961; RMSR 0.102; RMSEA .050; ECVI 0.328) y de acuerdo con las medidas de ajuste incremental y de parsimonia (ver Tablas $\mathrm{N} .{ }^{\circ} 8$ y 9 ) significativamente superior, al modelo independiente y muy similar al modelo saturado.

Tabla N. ${ }^{\circ} 7$ Medidas absolutas de ajuste para los modelos generados. Primer y segundo análisis factorial confirmatorio submuestras 1 y $2 ;{ }^{* * *} p<.01$

\begin{tabular}{lccccc}
\hline & \multicolumn{5}{c}{ Índice de Ajuste } \\
\cline { 2 - 6 } Modelo & $\square^{2}$ & GFI & RMSR & RMSEA & ECVI \\
\hline \multicolumn{5}{c}{ Primera solución factorial (submuestra 2) } \\
Independiente (13 ítems) & $4874.936^{\times \times x}$ & .395 & 0.891 & .245 & 4.791 \\
Saturado (13 ítems) & 0 & 1 & 0 & & 0.178 \\
3 factores 15 ítems & 711.25 & .912 & 0.188 & .084 & 0.760 \\
3 factores 13 ítems & $181.352^{\times \times *}$ & .973 & 0.085 & .043 & 0.234 \\
\hline
\end{tabular}




\begin{tabular}{lccccc}
\hline \multicolumn{5}{c}{ Segunda solución factorial (submuestra 1) } \\
Independiente (13 ítems) & $4916.27^{* * *}$ & .406 & 0.853 & .241 & 4.645 \\
Saturado (13 ítems) & 0 & 1 & 0 & & 0.171 \\
3 factores 13 ítems & $290.764^{* * *}$ & .961 & 0.102 & .050 & 0.328 \\
\hline
\end{tabular}

Tabla $\mathrm{N}^{\circ} 8$ Medidas de ajuste incremental para los modelos generados. Primer y segundo análisis factorial confirmatorio submuestras 1 y 2

\begin{tabular}{|c|c|c|c|c|}
\hline \multirow[b]{2}{*}{ Modelo } & \multicolumn{4}{|c|}{ Índice de Ajuste } \\
\hline & AGFI & TLI & NFI & $\mathrm{CFI}$ \\
\hline \multicolumn{5}{|c|}{ Primera solución factorial (submuestra 2) } \\
\hline Independiente (13 ítems) & .294 & 0 & 0 & 0 \\
\hline Saturado (13 ítems) & & & 1 & 1 \\
\hline 3 factores 15 ítems & .879 & .875 & .884 & .897 \\
\hline 3 factores 13 ítems & .961 & .969 & .963 & .975 \\
\hline \multicolumn{5}{|c|}{ Segunda solución factorial (submuestra 1) } \\
\hline Independiente (13 ítems) & .307 & 0 & 0 & 0 \\
\hline Saturado (13 ítems) & & & 1 & 1 \\
\hline 3 factores 13 ítems & .943 & .941 & .941 & .953 \\
\hline
\end{tabular}

Tabla N.$^{\circ} 9$ Medidas de ajuste de parsimonia para los modelos generados. Primer y segundo análisis factorial confirmatorio submuestras 1 y 2

\begin{tabular}{lcccc}
\hline & \multicolumn{5}{c}{ Índice de Ajuste } \\
\cline { 2 - 6 } Modelo & PNFI & PGFI & CMIN/GL & AIC \\
\hline \multicolumn{5}{c}{ Primera solución factorial (submuestra 2) } \\
Independiente (13 ítems) & 0 & .339 & 62.499 & 4900.936 \\
Saturado (13 ítems) & 0 & & & 182.000 \\
3 factores 15 ítems & .733 & .661 & 8.175 & 777.250 \\
3 factores 13 ítems & .763 & .663 & 2.925 & 239.352 \\
\hline \multicolumn{5}{c}{ Segunda solución factorial (submuestra 1) } \\
Independiente (13 ítems) & 0 & .348 & 63.029 & 4942.247 \\
Saturado (13 ítems) & 0 & & & 182.000 \\
3 factores 13 ítems & .748 & .655 & 4.69 & 348.764 \\
\hline
\end{tabular}


En la Figura N.$^{\circ} 1$ se presenta el modelo de medición para los 13 ítems agrupados en tres factores, incluyendo los coeficientes de regresión estandarizados entre ítems y factores y las saturaciones factoriales estandarizadas (comunalidades) de cada uno de los ítems.

Fig. N. ${ }^{\circ} 1$ Modelo de medición para la escala. Análisis factorial confirmatorio submuestra 2. Fuente: de los autores

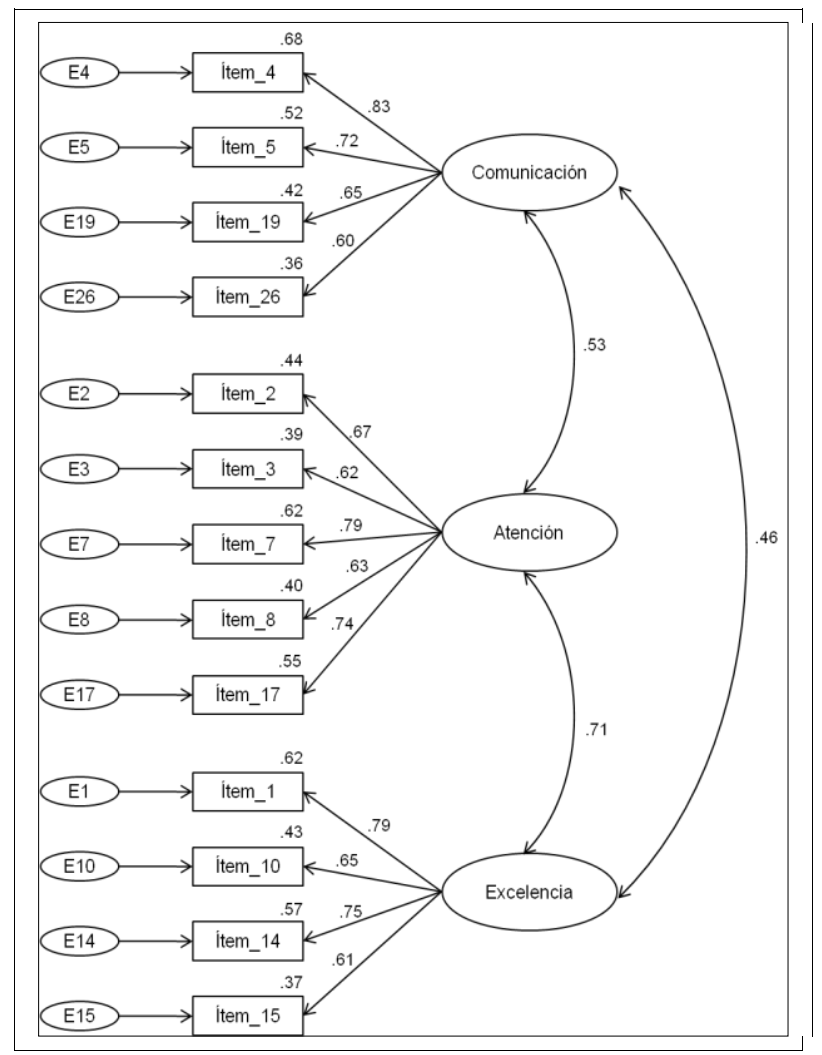

El primer factor: Comunicación, presenta, en general, saturaciones factoriales estandarizadas elevadas (mayores a .40), salvo en el caso del ítem 26 , con una saturación con el factor moderada (.36). En el segundo factor, Atención, todos los ítems resultan bien explicados a partir del constructo hipotetizado, como muestran una saturación mínima de .39 (ítem 3), y, el resto, por encima o igual a .40. Finalmente, el tercer factor, Excelencia, presenta una saturación factorial mínima de .37, correspondiente al ítem 15 mientras que el resto de ítems explicados por el factor presentan saturaciones por encima de 42 .

Por su parte, la Tabla N. ${ }^{\circ} 10$ ofrece la estimación de las correlaciones entre los tres factores de la escala. Todos los factores correlacionan entre sí de forma estadísticamente 
significativa $(\mathrm{p}<.01)$. Las correlaciones entre Comunicación y los factores Atención y Excelencia pueden considerarse moderadas, con valores de .533 y .456 respectivamente, mientras que la relación entre estos dos últimos factores es alta (.711), lo cual muestra que a medida que aumenta la autoeficacia percibida en alguno de los factores, también aumenta en los otros dos.

Tabla N. ${ }^{\circ} 10$ Correlaciones entre los tres factores de la escala. Primer análisis factorial confirmatorio submuestra 2

\begin{tabular}{lccc}
\hline \multicolumn{1}{c}{ Factor } & Comunicación & Atención & Excelencia \\
\hline Comunicación & & .533 & .456 \\
Atención & .533 & & .711 \\
Excelencia & .456 & .711 & \\
\hline
\end{tabular}

En la Figura $\mathrm{N} .^{\circ} 2$ se presenta el modelo de medición para los 13 ítems agrupados en tres factores, incluyendo los coeficientes de regresión estandarizados entre ítems y factores y las saturaciones factoriales estandarizadas (comunalidades) de cada uno de los ítems.

Fig. $\mathbf{N} \cdot{ }^{\circ} 2$ Modelo de medición para la escala. Análisis factorial confirmatorio submuestra 1. Fuente: de los autores

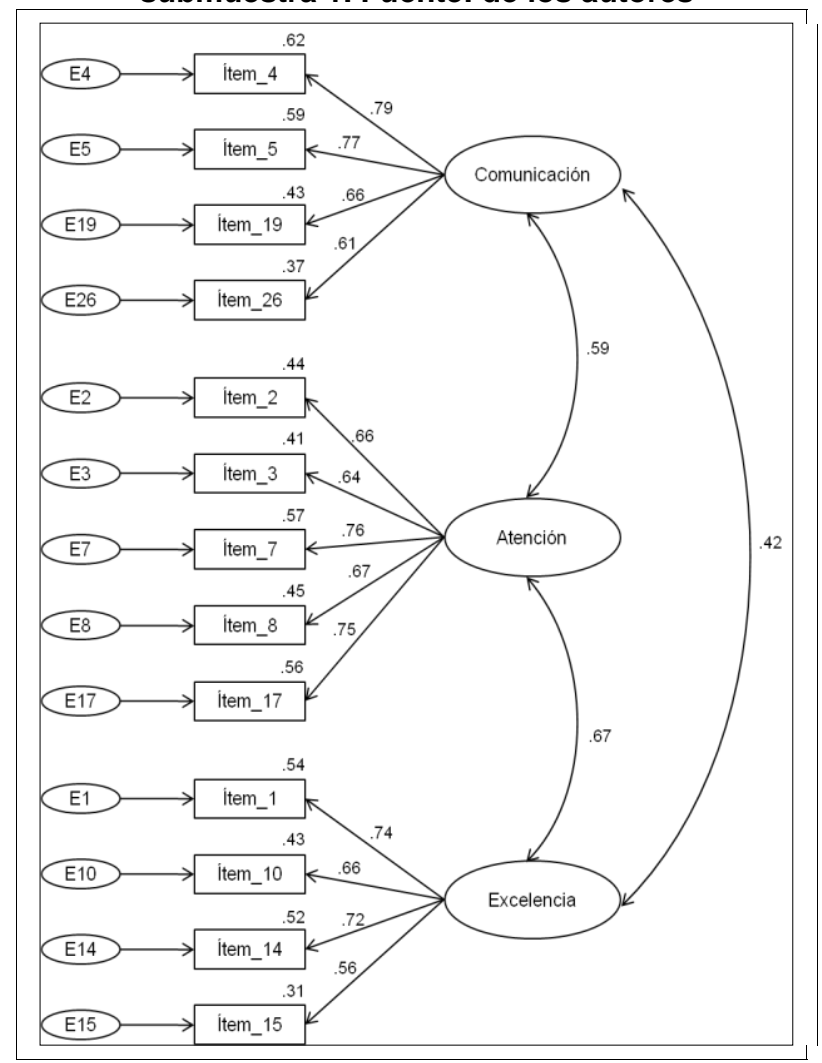


El primer factor: Comunicación, presenta, en general, saturaciones factoriales estandarizadas elevadas (mayores a .40), salvo en el caso del ítem 26, con una saturación con el factor moderada (.37). En el segundo factor, Atención, todos los ítems resultan bien explicados a partir del constructo hipotetizado, como muestran una saturación mínima de .41 (ítem 3) y el resto, por encima del .43. Finalmente, el tercer factor, Excelencia, presenta una saturación factorial baja de .31, correspondiente al ítem 15 mientras que el resto de ítems explicados por el factor presentan saturaciones por encima de .42 .

Por su parte, la Tabla $\mathrm{N} .^{\circ} 11$ ofrece la estimación de las correlaciones entre los tres factores de la escala. Todos los factores correlacionan entre sí de forma estadísticamente significativa $(\mathrm{p}<.01)$. Las correlaciones entre Comunicación y los factores Atención y Excelencia pueden considerarse moderadas, con valores de .588 y .422 respectivamente, mientras que la relación entre estos dos últimos factores es alta (.674), lo cual muestra que a medida que aumenta la autoeficacia percibida en alguno de los factores, también aumenta en los otros dos.

Tabla $\mathbf{N}^{\circ}$ 11. Correlaciones entre los tres factores de la escala. Segundo análisis factorial confirmatorio submuestra 1

\begin{tabular}{lccc}
\hline \multicolumn{1}{c}{ Factor } & Comunicación & Atención & Excelencia \\
\hline Comunicación & & .588 & .422 \\
Atención & .588 & & .674 \\
Excelencia & .422 & .674 & \\
\hline
\end{tabular}

\subsubsection{Congruencia entre los factores de las dos soluciones factoriales}

Los valores de los Coeficientes de Congruencia y de los Coeficientes de correlación de Pearson entre los pesos factoriales (coeficientes de regresión estandarizados) de los factores obtenidos en los análisis factoriales confirmatorios llevados a cabo con las submuestras 1 y 2; indican, de acuerdo con lo sugerido por Cureton y D'Agostino (1983), Mulaik (1972) y Cliff (1966), una alta congruencia entre pares de componentes (Tabla $N^{\circ} 12$ ). 
Tabla $N^{\circ} 12$. Coeficientes de Congruencia y de correlación de Pearson entre pesos factoriales. Análisis factoriales confirmatorios submuestras 1 y 2

\begin{tabular}{lcc}
\hline \multicolumn{1}{c}{ Factor } & $\begin{array}{c}\text { Coeficiente de } \\
\text { Congruencia }\end{array}$ & $\begin{array}{c}\text { Coeficiente de } \\
\text { correlación }\end{array}$ \\
\hline Comunicación & .999 & .929 \\
Atención & .999 & .965 \\
Excelencia & .999 & .948 \\
\hline
\end{tabular}

\subsubsection{Fiabilidad de las subescalas}

Las subescalas (constructos) resultantes en los análisis factoriales confirmatorios, de ambas submuestras, poseen fiabilidades superiores a .7, evidenciando, de acuerdo con Hair et al. (1999) una fiabilidad adecuada, particularmente si se considera el número reducido de ítems. En cuanto a la varianza extraída solo en el factor (constructo) Comunicación se logra el mínimo sugerido; en los factores Atención y Excelencia los valores están relativamente cerca de .5, valor mínimo sugerido para la medida de varianza extraída (Tabla №13).

Tabla $\mathbf{N}^{\circ}$ 13. Fiabilidad y varianza extraída de los constructos obtenidos en los análisis factoriales confirmatorios submuestras 1 y 2

\begin{tabular}{lccccc}
\hline \multirow{2}{*}{ Constructos } & \multicolumn{2}{c}{ Submuestra 1 } & & \multicolumn{2}{c}{ Submuestra 2 } \\
\cline { 2 - 3 } \cline { 5 - 6 } Comunicación & Fiabilidad & Varianza & & Fiabilidad & Varianza \\
Atención & .797 & .500 & & .800 & .502 \\
Excelencia & .822 & .482 & & .823 & .483 \\
\hline
\end{tabular}

3.2.5 Invarianza de la estructura factorial en las dos submuestras (validación cruzada)

Para analizar la invarianza de la estructura factorial de la escala se siguieron las recomendaciones de Herrero (2010) estimando el mismo modelo en ambas submuestras; habiéndose determinado como base el modelo trifactorial en 13 ítems antes descrito, se realizó un análisis de factores confirmatorio simultáneamente en las dos muestras forzando a la invarianza de la imponiendo la igualdad de cargas factoriales. 
En la Tabla N. ${ }^{\circ}$ 14, se presentan los resultados: el primer modelo no impone ninguna igualdad, mostrando índices de ajuste muy adecuados (GFI .969; RMSR 0.093; RMSEA .037; ECVI 0.247); en el segundo modelo se impone la igualdad de cargas factoriales mostrando índices de ajuste similares al modelo anterior (GFI .969; RMSR 0.096; RMSEA .036; ECVI 0.242). La comparación entre los modelos sugiere una misma estructura factorial para ambas submuestras en lo que se refiere a las saturaciones factoriales $X^{2}(10)=3.685$, $p=.96$.

Tabla $N^{\circ} 14$ Medidas absolutas de ajuste para los modelos que se comparan para la prueba de invarianza de la estructura factorial; ${ }^{* \star *} p<.01$

\begin{tabular}{lccccc}
\hline \multirow{2}{*}{ Modelo } & \multicolumn{5}{c}{ Índice de Ajuste } \\
\cline { 2 - 6 } & $\square^{2}$ & GFI & RMSR & RMSEA & ECVI \\
\hline Modelo Sin & $662.772^{* * *}$ & .969 & 0.093 & 0.037 & 0.247 \\
$\begin{array}{l}\text { Restricciones } \\
\text { Invarianza Cargas }\end{array}$ & $666.457^{* * *}$ & .969 & 0.096 & 0.036 & 0.242 \\
\hline Factoriales & & & & & \\
\hline
\end{tabular}

\section{Discusión y Conclusiones}

El Análisis factorial exploratorio del instrumento autoeficacia en conductas académicas llevado a cabo en cada una de las submuestras estudiadas puso de manifiesto una estructura factorial de tres componentes: Comunicación, Atención y Excelencia, llevando, además, a eliminar 12 de los 27 ítems analizados. Los componentes de ambas submuestras evidenciaron una consistencia interna adecuada, así como una alta congruencia entre pares de componentes, particularmente si se considera el número reducido de ítems en cada uno de ellos.

Por su parte, el Análisis Factorial Confirmatorio indicó que el modelo de medición de 15 ítems en tres factores ajusta aceptablemente, pero no de manera óptima; un examen de los coeficientes de regresión estandarizados de los ítems con cada uno de sus factores latentes permitió identificar que, retirando dos ítems más, los índices mejoran de manera considerable y el ajuste de los datos al modelo teórico de 13 ítems agrupados en tres factores es óptimo. Al mismo tiempo que los tres factores así obtenidos presentan en general saturaciones factoriales estandarizadas adecuadas. De igual modo, los tres factores correlacionan entre sí de forma positiva y estadísticamente significativa lo cual muestra que a 
medida que aumenta la autoeficacia percibida en alguno de los factores, también aumenta en los otros dos. Conjuntamente con todo lo antes dicho, los valores de los coeficientes de congruencia y de los coeficientes de correlación de Pearson entre los pesos factoriales (coeficientes de regresión estandarizados) de los factores obtenidos en los análisis factoriales confirmatorios llevados a cabo con las submuestras 1 y 2, así como los resultados del análisis de la invarianza de la estructura factorial entre las submuetras indican una alta congruencia entre pares de componentes. Lo que sugiere la existencia de fuertes evidencias de la validación cruzada de la medida y, por tanto, de la estabilidad de la estructura, hasta que no se demuestre lo contrario.

En síntesis, el análisis de las propiedades psicométricas ha mostrado que una estructura trifactorial es viable y adecuada de acuerdo con los requisitos psicométricos establecidos cuando los informantes son los propios alumnos. La estructura de tres factores, atendiendo a criterios estadísticos y sustantivos, ha evidenciado adecuados indicadores de ajuste, de fiabilidad y de validez. Sin embargo, y como ha sido indicado por diferentes investigadores, la validez factorial de los instrumentos de medida debe ser demostrada con muestras que presenten diferencias tanto poblacionales como culturales, de tal manera que, consideramos que más estudios son necesarios con el fin de corroborar o refutar los datos obtenidos en la presente investigación.

\section{Aportes del presente trabajo al diseño de escalas de autoeficacia}

En las escalas de autoeficacia habitualmente se les pregunta a las personas acerca de sus capacidades operativas en el presente, no acerca de sus capacidades potenciales o sobre sus capacidades futuras esperadas (Bandura, 2001). En el diseño de los instrumentos empleados en nuestra investigación para medir autoeficacia, además de que el sujeto responde sobre la frecuencia con que actualmente realiza una determinada conducta o se percibe con determinada característica; responde también en forma ideal y si se esfuerza en cambiar, con qué frecuencia realizaría o manifestaría dichas acciones y/o habilidades. Esto es, cada sujeto responde a los ítems del instrumento en tres escenarios distintos: Escenario actual, respondiendo en el contexto: actualmente con qué frecuencia realiza determinada conducta o se percibe con determinada característica. Escenario ideal, respondiendo en el contexto: con qué frecuencia desearía realizar determinada conducta o percibirse con determinada característica. Escenario de cambio, respondiendo en el contexto: si me 
esfuerzo en cambiar con que frecuencia realizaría determinada conducta o me percibiría con determinada característica.

Para luego a partir de sus respuestas obtener 7 índices: 1) Autoeficacia percibida actualmente.- obtenida a partir de las respuestas al escenario actual. 2) Autoeficacia deseada.- obtenida a partir de las respuestas al escenario ideal. 3) Autoeficacia alcanzable en el futuro.- obtenida a partir de las respuestas al escenario de cambio. 4) Grado de insatisfacción o disonancia en la autoeficacia percibida.- obtenida a través de la diferencia entre el índice 2 y 1 (ideal menos actual). 5) Posibilidad de mejoría en la autoeficacia percibida.- obtenida a través de la diferencia entre el índice 3 y 1 (cambio menos actual). 6) Proporción de autoeficacia percibida en relación a la deseada.- obtenida a partir del índice 1 y 2 (actual entre ideal por 100) y 7) Proporción de autoeficacia percibida en relación a la alcanzable.- obtenida a partir del índice 1 y 3 (actual entre cambio por 100).

Esto permite, entre otras cosas, generar disonancia cognoscitiva en el sujeto en relación con su autoeficacia percibida en el presente, el nivel de autoeficacia deseado o ideal, y la posibilidad de mejoría en la autoeficacia percibida. La disonancia cognoscitiva hace referencia a la tensión o desarmonía interna del sistema de ideas, creencias, emociones y actitudes (cogniciones) que percibe una persona al mantener al mismo tiempo dos pensamientos que están en conflicto, o por un comportamiento que entra en conflicto con sus creencias (Festinger, 1957). En cuanto hace su aparición, la disonancia brota una fuerza igual y de signo contrario para reducirla; en otras palabras, la disonancia actúa del mismo modo que un impulso, necesidad o estado de tensión (Ovejero, 1993). La presencia de la disonancia lleva a una acción para reducirla de idéntica forma que, por ejemplo, el hambre lleva a una acción para evitarla.

\section{Referencias}

Arbuckle, James. (2007). AMOS users guide version 16.0.

Bagozzi, Richard y Baumgartner, Hans. (1994). The evaluation of structural equation models and hypothesis testing. En Principles of Marketing Research (cap. 10, 386-419). Brasil: Blackwell Itd.

Bandura, Albert. (1982). Self-efficacy mechanism inhuman agency. American Psychologist, 37(2) 122-147. 
Bandura, Albert. (1986). Pensamiento y acción. Fundamentos sociales. Madrid: Alianza.

Bandura, Aalbert. (1995) Exercise of personal and collective efficacy. En: Bandura, Albert.

(Ed.) Self - efficacy in Changing Societies. EEUU: University of Cambridge.

Bandura, Albert. (1997). Self-efficacy: The exercise of Control. New York: Freeman.

Bandura, Albert. (2000) Self-efficacy: the foundation of agency. En Walter. Perrig (Ed.): Control of human behavior, mental processes and consciousness (pp.17-33). N. J: Erlbaum.

Bandura, Albert. (2001). Guía para la construcción de escalas de autoeficacia. Recuperado de: http://www.revistaevaluar.com.ar/effguideSpanish.htm

Batista, Joan y Coenders, Germá. (2000). Modelos de ecuaciones estructurales. Cuadernos de estadística, 6. Madrid: La Muralla.

Blanco, Humberto; Ornelas, Martha; Beltrán, Francisco; Mondaca, Fernando; Rodríguez, Judith et al. (2007). Editor para la construcción y aplicación de escalas por medio de una PC. Tecnociencia Chihuahua, 1(1), 55-59.

Bong, Mimi. (2001). Role of self-efficacy and task-value in predicting college students course performance and future enrollment intentions. Contemporary Educational Psychology, 26, 553-570.

Catena, Andrés; Ramos, Manuel y Trujillo, Humberto. (2003). Análisis multivariado, un manual para investigadores. Madrid: Ed. Biblioteca Nueva

Cattell, Raymond. (1966). The meaning and strategic use of factor analysis. En Raymond. Cattell (Ed.), Handbook of Multivariate Experimental Psychology. Chicago: Rand McNally.

Choi, Namok. (2004). Sex role group differences in specific, academic, and general selfefficacy. Journal of Psychology, 138, 149-159.

Cliff, Norman. (1966). Orthogonal rotation to congruence. Psychometrika, 31, 33-42

Costello, Anna y Osborne, Jason. (2005). Best practices in exploratory factor analysis: four recommendations for getting the most from your analysis. Practical Assessment, Research and Evaluation, 10(7), 1-9.

Cureton, Edward y D'Agostino, Ralph. (1983). Factor Analysis: An applied approach. London: Lawrence Erlbaum Associates.

Festinger, Leòn. (1957). A theory of cognitive dissonance. Stanford, CA: Stanford University Press. 
Hackett, Gail. (1995). Self-efficacy in career choice and development. En Albert. Bandura (Ed.) Self-efficacy in changing societies (pp. 232-258). New York: Cambridge University Press.

Hair Joseph; Anderson, Rolph; Tatham Ronald y Black, William. (1999). Análisis multivariante de datos. México: Ed. Prentice Hall.

Herrero, Juan. (2010). El análisis factorial confirmatorio en el estudio de la estructura y estabilidad de los instrumentos de evaluación: un ejemplo con el cuestionario de autoestima CA-14. Intervención Psicosocial, 19, 3, 289-300.

Hu, Li-tze y Bentler, Peter. (1995). Evaluating model fit. In Rick. Hoyle (Ed.), Structural equation modelling: Concepts, issues and applications (pp. 76-99). Thousand Oaks, C A: Sage Publications.

Huertas, Juan. (1997). Motivación. Querer aprender. Buenos Aires: Aique.

Kaiser, Henry. (1958). The Varimax criterion for analytical rotation in factor analysis. Psychometrika, 23, 187-200.

Leighton, Jacqueline; Gokiert, Rebecca y Cui, Ying. (2007). Using Exploratory and Confirmatory Methods to Identify the Cognitive Dimensions In a Large-Scale Science Assessment. International Journal of Testing, 7(2), 141-189.

Luszczynska, Aleksandra; Gibbons, Frederick; Piko, Bettina y Tekozel, Mert. (2004). Selfregulatory cognitions, social comparison, perceived peers' behaviors as predictors of nutrition and physical activity: A comparison among adolescents in Hungary, Poland, Turkey, and USA. Psychology and Health, 19, 577-593.

Luszczynska, Aleksandra; Scholz, Urte y Schwarzer, Ralf. (2005). The general self-efficacy scale: Multicultural validation studies. The Journal of Psychology, 139(5), 439-457.

Mulaik, Stanley. (1972). The foundations of factor analysis. New York: McGraw-Hill.

Muñiz, José. (1998). Teoría clásica de los test. Madrid: Pirámide.

Nunnally, Jum y Bernstein, Ira. (1995). Teoría Psicométrica. México: McGraw-Hill.

Ovejero, Anastasio. (1993). La teoría de la disonancia cognoscitiva. Psicothema, 5, 001.

Pajares, Frank. (1996). Current Directions in Self Research: Self-efficacy. Ponencia presentada en el Annual Meeting of the American Educational Research Association, New York, April.

Pajares, Frank. (2001). Self-efficacy beliefs in academic settings. Review of Educational Research, 66(4), 543-578. 
Pajares, Frank. (2002a). Overview of Social Cognitive Theory and Self - Efficacy. EEUU: Emory University. Recuperado de http://www.des.emory.edu/mfp/eff.html

Pajares, Frank. (2002b). Self-efficacy beliefs in academic context: an outline. Recuperado de http://www.des.emory.edu/mfp/efftalk.html

Pajares, Frank; Hartley, James y Valiante, Giovanni. (2001). Response format in Writing selfefficacy assessment: greater discrimination increases prediction. Measurement and evaluation in counseling and development. 33(4), 214-221.

Pajares, Frank y Schunk, Dale. (2001). Self-Beliefs and School Success: Self-Efficacy, SelfConcept, and School Achievement. In R. Riding y S. Rayner (Eds.), Perception (pp. 239-266). London: Ablex Publishing.

Pastorelli, Concetta; Caprara, Gian; Barbaranelli, Claudio; Rola Jarek; Rozsa, Sandor y Bandura, Albert . (2001). The estructure of children's perceived self-efficacy: A cross national study. European Journal of Psychological Assessment, 17(2) 87-97.

Prieto Navarro, Leonor (2003). La autoeficacia en el contexto académico. Exploración bibliográfica comentada. Recuperado de http://www.des.emory.edu/mfp/prieto.pdf

Pintrich, Paul; Cross, David; Kozma, Robert y McKeachie, Wilbert. (1986). Instructional psycology. Annual Review of Psychology, 37, 611-651.

Pintrich, Paul y De Groot, Elisabeth. (1990). Motivational and self-regulated learning components of classroom academic performance. Journal of educational psychology, 82, 1, 33-40.

Pintrich, Paul y García, Teresa. (1993). Intraindividual differences in students' motivation and selfregulated learning. German journal of educational psichology, 7(3), 99-107.

Schunk, Dale. (1989). Self-efficacy and cognitive skill learning. En C. Ames y R. Ames (Eds.). Research on motivation in education: Vol. 3: Goals and cognitions (pp. 13-44). San Diego: Academic Press.

Schunk, Dale y Zimmerman, Barry. (1997). Developing self-efficacious readers and writers: the role of social and self-regulatory processes. Guthrie, J. y Wigfield, A. (Eds.) Reading engagement: motivation readers through integrated instruction (pp.34-50). Newark, DE: International Reading Association.

Thompson, Bruce. (2004). Exploratory and Confirmatory Factor Analysis. Understanding concepts and applications. Washington, D C: American Psychological Association.

Valiante, Giovanni. (2000). Writing Self-efficacy and gender orientation: A developmental perspective, a dissertation proposal. Atlanta: Emory University.

Weinstein, Richard. (1989). Perceptions of classroom processes and student motivation: Children's views of self-fulling prophecies. En C. Ames y R. Ames (Eds.). Research on 
motivation in education: Vol. 3: Goals and cognitions (pp. 187-221). San Diego: Academic Press.

Wolters, Christopher. (2004). Advancing achievement goal theory: using goal structures and goal orientations to predict students motivation, cognition and achievement. Journal of Educational Psychology, 96(2), 236-250.

Zimmerman, Barry. (1990). Self-regulating academic learning and achievement: The emergence of a social cognitive perspective. Educational Psychology Review, 2, 173-201.

Zimmerman, Barry. (1995). Self-efficacy and educational development. En A. Bandura (Ed.). Self-efficacy in changing societies (pp. 202-231). New York: Cambridge University Press.

Zimmerman, Barry y Kitsantas, Anastasia. (2005). Homework practice and academic achievement. The mediating role of self-efficacy and perceived responsibility beliefs. Contemporany Educational Psychology, 30(4) 397-417. doi: 10.1016/j.cedpsych.2005.05.003 